\title{
Espace virtuel interconnecté et Théâtre (2). Influences sur le jeu scénique.
}

\author{
Interconnected virtual space and Theater. Influences on acting.
}

\author{
Georges Gagneré ${ }^{1}$, Cédric Plessiet ${ }^{2}$ \\ 1 EA 1573, Université Paris VIII, Saint-Denis, France, georges.gagnere@univ-paris8.fr \\ ${ }^{2}$ INREV EA 4010, Université Paris VIII, Saint-Denis, France, cedric.plessiet@univ-paris8.fr
}

\begin{abstract}
RÉSUMÉ. L'article expose la suite d'une recherche-création sur le jeu scénique théâtral et la direction d'avatar. II décrit tout d'abord le changement de paradigme induit par le passage du medium vidéo à l'image en 3D pour transférer une présence scénique dans espace virtuel à l'aide d'un dispositif de capture de mouvement inertiel. II s'appuie sur un cas d'usage pour expliquer la nouvelle approche nécessaire à la fois en terme de jeu et de direction. II conclue par l'exposition de perspectives synthétisant les résultats obtenus et exposant une feuille de route des explorations à venir.

ABSTRACT. The article presents the second step of a practise based research about theatrical acting and avatar direction. It first describes the paradigm shift induced by the move from video medium towards 3D image for transferring scenic presence in virtual space using an inertial motion capture device. It takes a use case for explaining the new approach needed both in acting and direction issues. It ends with a synthese of the achieved results and prospects about new explorations.

MOTS-CLÉS. Capture de mouvement, direction d'avatar, jeu augmenté, présence scénique, réalité mixte, théâtre.

KEYWORDS. Augmented acting, avatar direction, theatrical liveness, mixed reality, motion capture, performing arts.
\end{abstract}

\section{Introduction}

Alors que le réseau est devenu omniprésent dans nos vies quotidiennes, l'impact qu'il peut avoir sur une scène de théâtre est encore assez peu étudié, notamment concernant les techniques de jeu scénique. Nous avions amorcée une première réflexion sur les outils informatiques nécessaires à mettre en place pour permettre à des acteurs et des actrices de théâtre d'habiter une scène mixte impliquant un espace virtuel en 3D en utilisant des dispositifs de capture de mouvement inertiels en wifi [GAG 18a, SAL 18]. Nous poursuivons ici cette réflexion en l'orientant vers une approche de la nature de l'avatar [AMA 13], de la qualité de sa présence scénique [FDI 11] ainsi que de sa plausibilité en tant que personnage [SLA 09].

Nous aborderons tout d'abord les moyens théâtraux actuellement employés, avec l'usage du medium vidéo, pour projeter une présence scénique dans une image 2D. Nous étudierons notamment l'exemple des dispositifs de téléprésence et de Pepper's Ghost pour illustrer le processus d'individuation intermédiale caractérisant l'évolution du jeu théâtral [PLU 11]. Nous expliquerons ensuite en quoi l'usage du réseau et de la réalité virtuelle en 3D provoque un changement de paradigme pour le jeu scénique et conduit à utiliser de nouveaux moyens pour capter la présence et la transférer dans l'espace 3D en utilisant des avatars.

Nous détaillerons les conséquences de ce changement de paradigme sur la relation entre les personnes physiques contrôleuses et l'avatar 3D contrôlé pour garantir la qualité du transfert de présence. Puis nous étudierons un cas d'usage détaillant le processus de direction scénique simultanée des personnes physiques et des avatars. Nous détaillerons au passage la technique de direction d'avatar utilisée avec le module AKN_Regie implémenté au sein d'un moteur de jeu vidéo, ce qui nous permettra d'expliciter la notion de position de référence virtuelle. Nous confluerons enfin par des perspectives résumant de nouvelles approches du jeu scénique qui nécessiteront l'ouverture de futurs chantiers d'expérimentation. 


\section{Tentatives d'habitation du virtuel par l'usage de l'image vidéo}

Jusqu'à présent, la médiatisation des acteurs et des actrices dans des dispositifs virtuels de spectacle vivant a eu essentiellement recours à l'utilisation du médium vidéo pour véhiculer la présence des corps physiques [DIX 07] [SAL 10], ce qui induit une situation de confrontation aux écrans et images vidéo [FER 18].

Nous partirons d'exemples de dispositifs existants recourant à la vidéo, la téléprésence et le Pepper's Ghost, que nous mettrons en correspondance avec le dispositif expérimental AvatarStaging, recourant à des images en 3D, afin de circonscrire les difficultés rencontrées pour habiter les images virtuelles.

\subsection{L’image vidéo 2D dans un dispositif de téléprésence}

Schématiquement, un dispositif de téléprésence confronte sur une scène un acteur à l'image vidéo temps réel d'un autre acteur physiquement distant. Il met ainsi en œuvre un équipement permettant une connexion temps réel entre les participants d'au moins deux espaces éloignés (cf. figure 1). Cela permet d'explorer de nouvelles formes scéniques [STA 10] qui reposent généralement sur l'infrastructure internet haut débit en déploiement croissant. Nous nous intéressons au fait que la présence scénique d'une partie des acteurs est rendue possible par la projection d'une image vidéo utilisant une capture en temps réel par une caméra, souvent augmentée par des effets visuels.

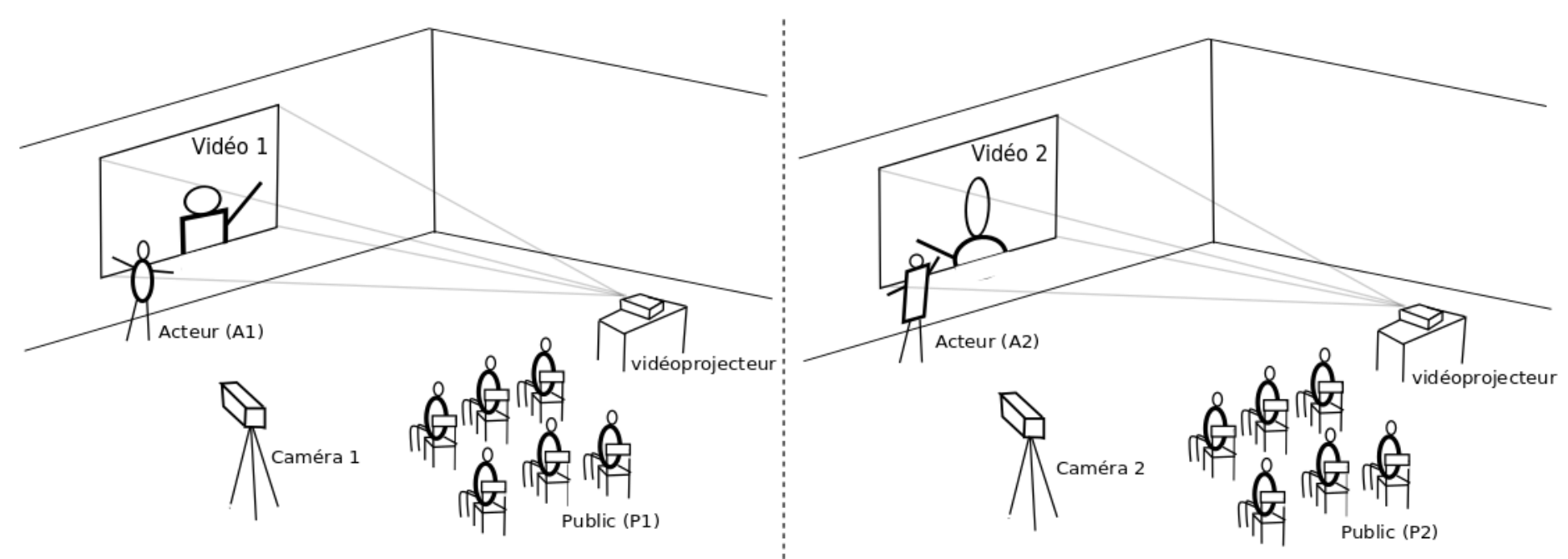

Figure 1. Dispositif de téléprésence

À gauche de la figure 1, la caméra 1 capture la présence de l'acteur A1, qui joue pour le public P1 en dialogue avec l'image de l'acteur distante Vidéo1. Dans l'espace symétrique distant à droite, on retrouve l'acteur de Vidéo1 en chair en os en A2, alors que l'acteur A1 est médiatisé à travers Vidéo2, chaque acteur dispose simultanément de deux registres de présence face au public, physique ou médiatisée par une captation vidéo. Chaque registre dispose de règles spécifiques, parfois contradictoires pour garantir la meilleure qualité de présence. Par exemple, si A1 veut donner l'impression de parler à $\mathrm{P} 2$, il devra regarder la caméra afin que Vidéo2 semble regarder le public, ce faisant il interrompt la relation avec son public P1. Étant donnée la symétrie des espaces, A1 peut imaginer sa manière d'être présent dans l'espace distant en observant l'image Vidéo2.

Les acteurs recourent à des techniques de jeu qui s'inspirent du jeu face à la caméra comme il se pratique au cinéma, en travaillant leur exposition au point focal de la caméra, et ils sont en dialogue avec un aplat vidéo qui joue comme une facette de la scénographie, impénétrable, sinon en y insérant sa propre image vidéo. Les metteurs en scène utilisent en effet souvent la possibilité de faire se rencontrer les deux acteurs en mixant les images vidéo1 et vidéo2. Mais il s'agit alors de superposer deux images dont la nature essentielle du contenu est le résultat d'une exposition face à une caméra. 


\subsection{Le subterfuge du reflet vidéo}

Un subterfuge souvent utilisé pour donner l'impression qu'une image vidéo augmente en profondeur une scénographie est de recréer une image en miroir de la scène et des comédiens (cf. figure 2).

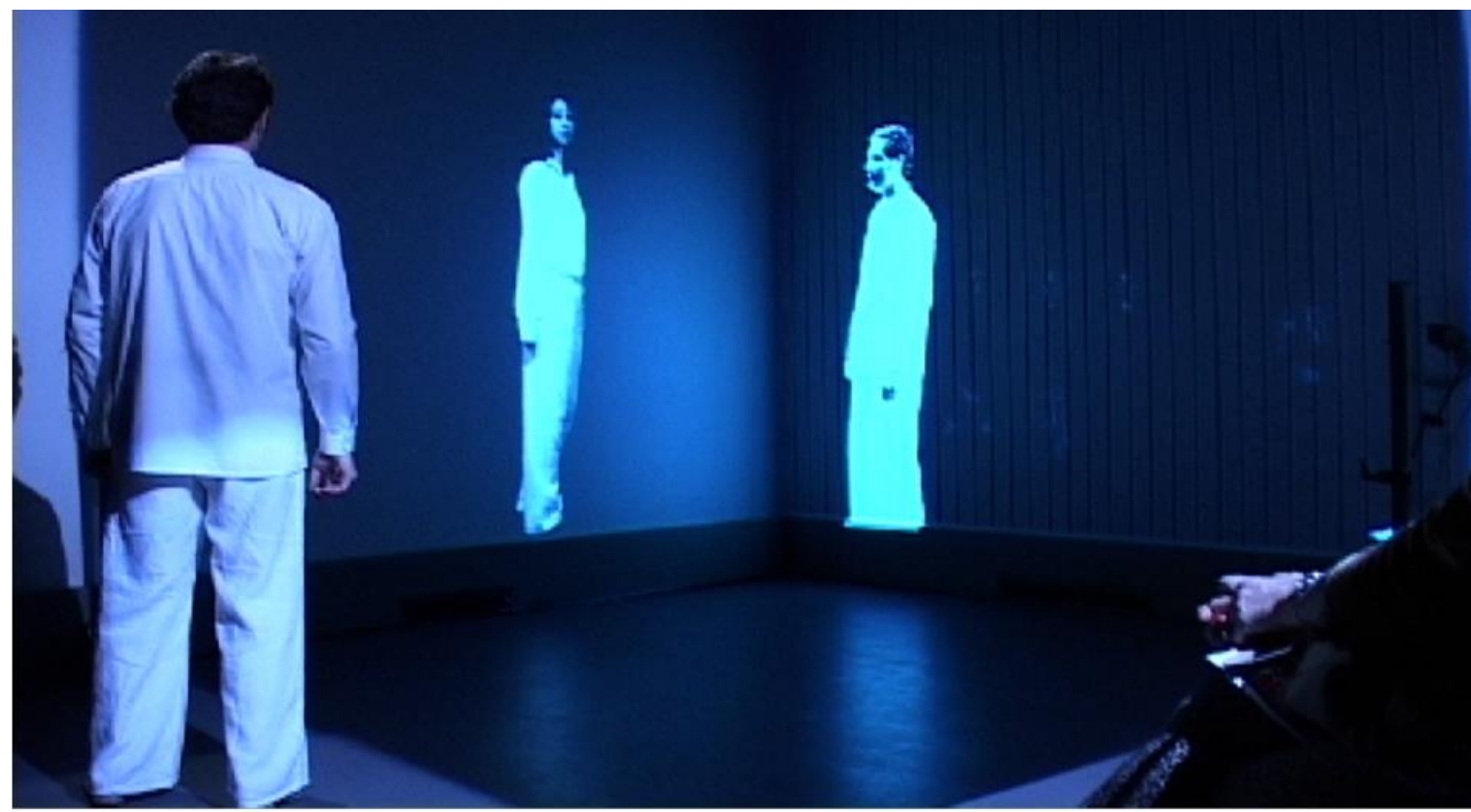

Figure 2. Dispositif de reflet vidéo.

L'acteur à gauche dans la figure semble se refléter dans un miroir, le faux reflet étant produit par la captation d'une caméra située sur le côté et dont les images sont vidéoprojetées sur un mur blanc. Le public peut alors imaginer qu'il y a un espace de l'autre côté du mur, à l'intérieur de l'image vidéo. Cette image pourrait aussi être peuplée d'autres images de personnages avec lesquels l'image-reflet de l'acteur physique interagirait. Par exemple, dans la figure 2, l'acteur semble s'adresser à une comédienne située dans un espace similaire derrière le mur mitoyen.

Dans le spectacle dont l'image est tirée [GAG 05], cette comédienne est préenregistrée. Mais elle aurait pu être filmée en direct dans un espace scénique symétrique éloigné. Nous serions dans un dispositif de téléprésence plus complexe que celui de la figure 1 . On jouerait alors avec le subterfuge de dédoubler l'espace physique par des espaces virtuels. Ce type de simulation implique cependant de fortes contraintes scéniques qui demandent aux artistes de se positionner avant tout face à une caméra qui ne capture qu'un point de vue retransmis en 2D. Il est souvent difficile pour la personne de se regarder directement pour voir l'effet produit et d'ajuster les actions scéniques. Le metteur en scène est d'un appui indispensable pour aider à fixer les positions dans l'espace physique qui permettront d'obtenir les adresses scéniques souhaitées dans l'image vidéo (comme c'est le cas dans la figure 2 où les deux acteurs, l'un physique, l'autre en reflet vidéo, regarde la même actrice virtuelle).

\subsection{Tentative d'hybridation entre physique et virtuel dans un dispositif de Pepper's Ghost}

Un autre subterfuge consiste à insérer une image dans la scénographie grâce à un écran semiréfléchissant, ainsi que le permettent le dispositif de Pepper's Ghost (cf. figure 3). L'image vidéo d'un acteur est projetée sur un écran situé au-dessus d'un écran transparent semi-réfléchissant incliné à $45^{\circ}$ situé sur la scène. Pour le public, l'acteur physique vu à travers l'écran, et l'acteur fantôme qui se réfléchit sur l'écran semi-transparent semblent exister dans le même espace scénique qui constitue alors une sorte de réalité en 2D 1/2, notion parfois utilisée quand deux images 2D sont juxtaposées dans l'espace. Le contexte de jeu est très compliqué pour l'acteur physique, car il ne voit pas son partenaire fantôme et joue dans le vide. 


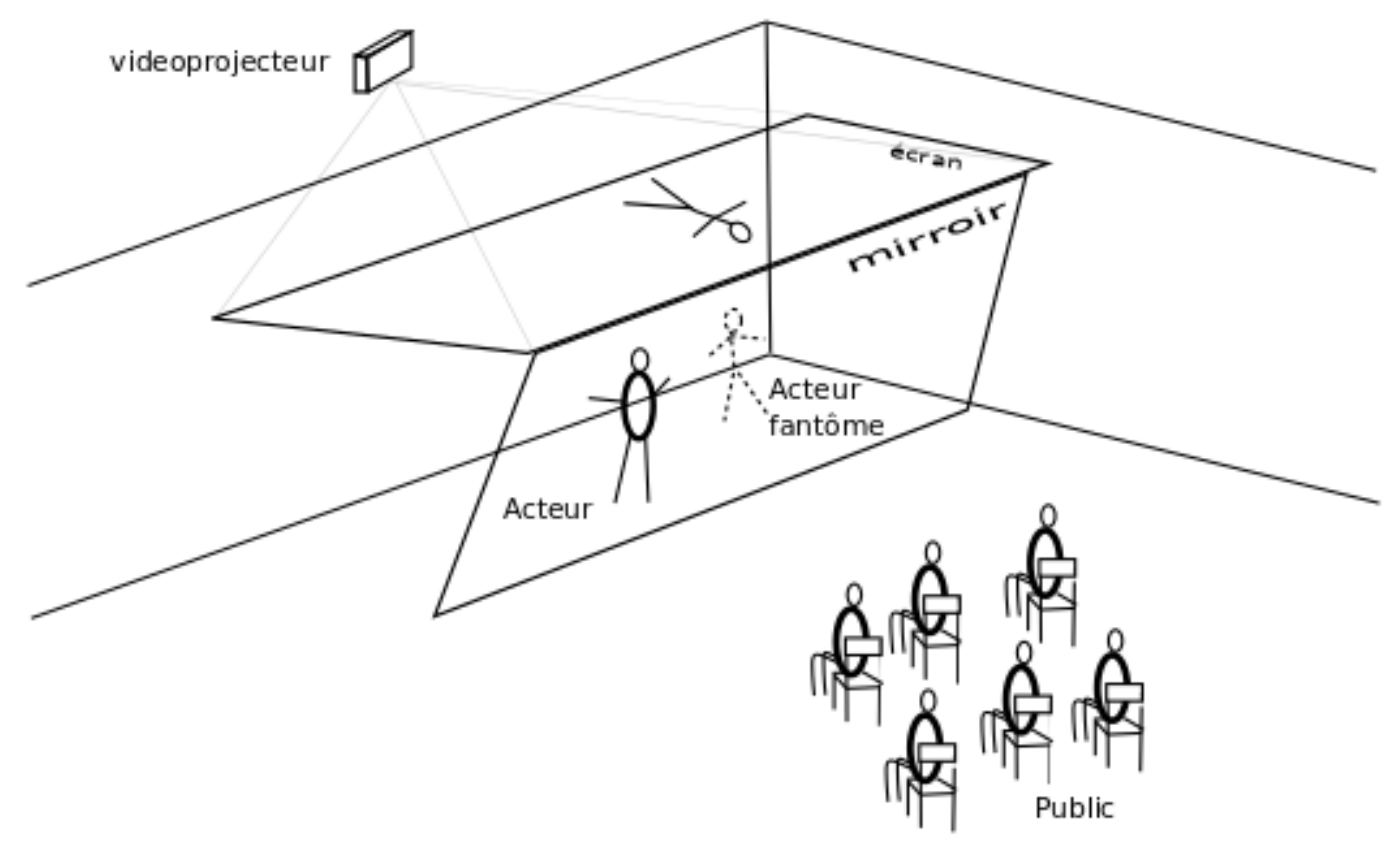

Figure 3. Dispositif de Pepper's ghost.

Comme dans l'effet miroir précédemment cité, l'intérêt est de pouvoir réaliser des effets visuels dont la magie illusionne le public. Dans ce dispositif, l'acteur physique n'a aucun moyen de jouer spatialement avec son partenaire, sinon par des effets de superposition compliqués à réaliser et dont l'efficacité visuelle est parfois hasardeuse. L'insertion d'une image 2D dans un espace physique ne facilite pas les interactions entre les deux acteurs, dont la réussite est pourtant un gage de qualité de leur présence scénique. La direction de l'acteur physique par le metteur en scène est similaire aux cas précédents, mais souvent encore plus difficile à réaliser, car l'acteur est aveuglé par les lumières latérales qui garantissent son éclairement, et donc sa visibilité à travers l'écran.

\section{Changement de paradigme introduit par l'interconnexion avec l'image 3D}

\subsection{L'image 3D n'est pas un médium vidéo}

La nature d'une image de synthèse en 3D produit un changement de paradigme dans la manière de penser la relation d'un acteur aux images. Une image virtuelle en 3D est générée en simulant la plupart des phénomènes qui se passent dans le monde physique. C'est aussi le cas lorsqu'on veut introduire une reproduction anthropomorphe, qu'on appellera dans notre cas avatar. Un avatar est informatiquement basé sur une sculpture matrice, c'est-à-dire une modélisation sous forme de maillage 3D doté d'un ensemble de mécanismes permettant de la manipuler pour la mettre en position, et qui donc dispose d'une potentialité de mouvements. Cette sculpture matrice est donc équipée d'un système d'actuation de mouvements, dont les sources peuvent avoir plusieurs origines : un animateur de jeu vidéo, une combinaison de capture de mouvement, un algorithme génétique. un acteur ne peut donc pas se projeter dans une image 3D par le médium vidéo. Si il souhaite habiter une sculpture matrice, il doit lui transmettre les mouvements qu'il réalise dans leur dimension spatiale. 


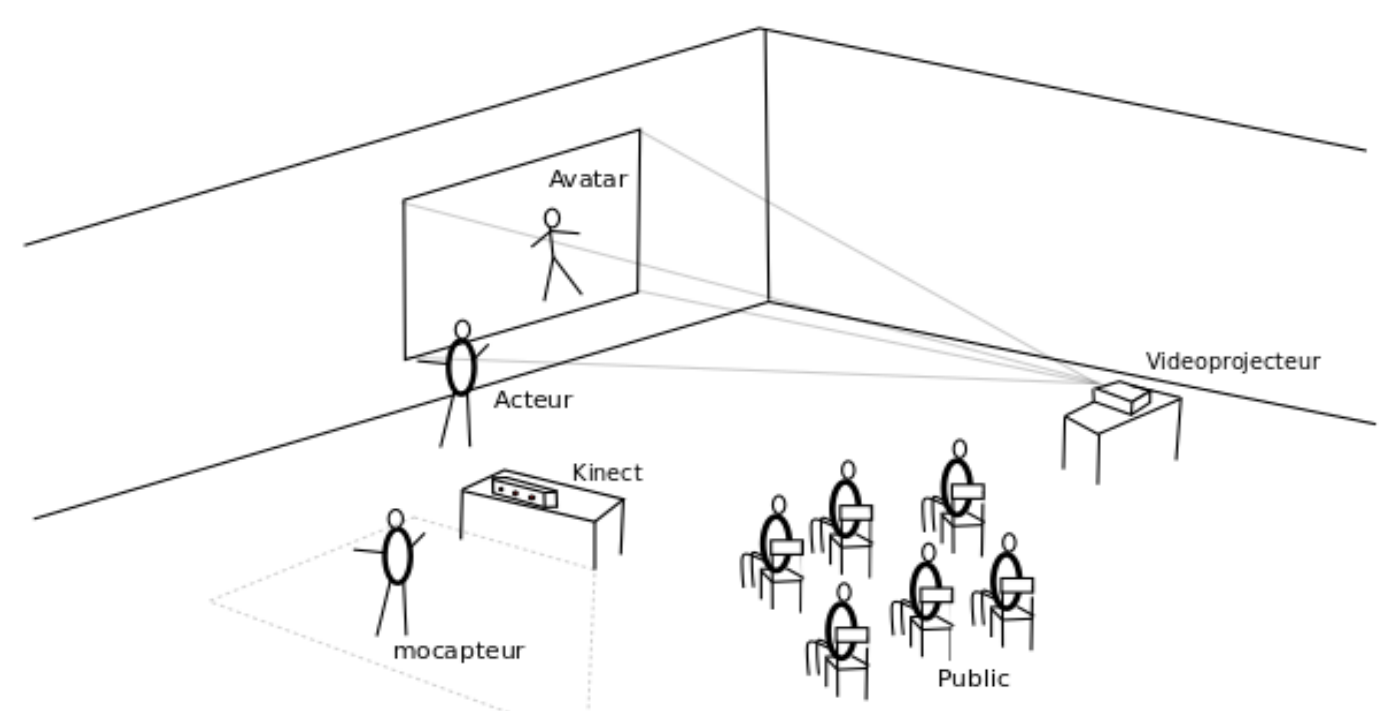

Figure 4. Dispositif AvatarStaging avec Kinect

C'est l'objet du dispositif expérimental AvatarStaging que nous avons mis en place pour nos expérimentations scéniques (cf. figure 4). Nous voyons ainsi à gauche le jeu d'une personne devant une caméra spéciale, la Kinect (fabriquée par Microsoft), qui permet de capturer les mouvements en 3D. Nous appellerons mocapteur la personne qui se place dans un dispositif de capture de mouvement en vue d'animer un avatar.

\subsection{Le recours à la motion capture en temps réel pour habiter un espace 3D}

Il ne s'agit donc plus d'exposer le corps de l'acteur au point de vue d'une caméra qui restituera une présence sur une surface, il faut être en mesure de capter la profondeur du corps pour faire exister un avatar en 3D. La Kinect a été le premier dispositif que nous avons utilisé au début de nos expérimentations. Il est intéressant de remarquer que la conception du dispositif est la parfaite illustration du changement de paradigme du passage de la vidéo à la 3D dans le domaine de la captation de la présence.

La Kinect est en effet constituée d'une caméra traditionnelle qui capte les couleurs, et d'une caméra infrarouge qui capte la réflexion de multiples faisceaux infrarouges balayant l'espace, ce qui permet d'établir une carte de profondeur. La caméra traditionnelle permet d'identifier la silhouette d'une personne, et son association avec la carte de profondeur permet de reconstruire algorithmiquement un squelette en 3D d'actuateurs permettant d'animer la sculpture matrice formant l'enveloppe de l'avatar. Pour le grand public, la Kinect a donc enrichi l'approche vidéo du corps en lui adjoignant une profondeur, lui ouvrant ainsi l'accès à l'exploration d'un espace 3D.

Nous avons cependant rapidement basculé vers une autre approche 3D du corps humain, indépendante des techniques vidéo car utilisant un système de repérage des parties du corps à partir d'accéléromètres physiquement placés sur les parties en question. Ce type de système a récemment évolué vers l'envoi direct sur le réseau en wifi des informations de chaque accéléromètre de manière indépendante. On utilise donc en quelque sorte un étiquetage des membres qui permet une reconstruction algorithmique du corps humain en temps réel.

\subsection{AvatarStaging et la capture de mouvement inertielle en wifi}

La version du dispositif d'AvatarStaging que nous utilisons ici pour illustrer notre réflexion implique l'utilisation d'un périphérique de capture de mouvement inertiel et géolocalisé, qui consiste à 
mettre en relation des performeurs physiques et des avatars dans une scénographie mixte juxtaposant une scène physique et une image 3D (cf. figure 5) [PLE 18].

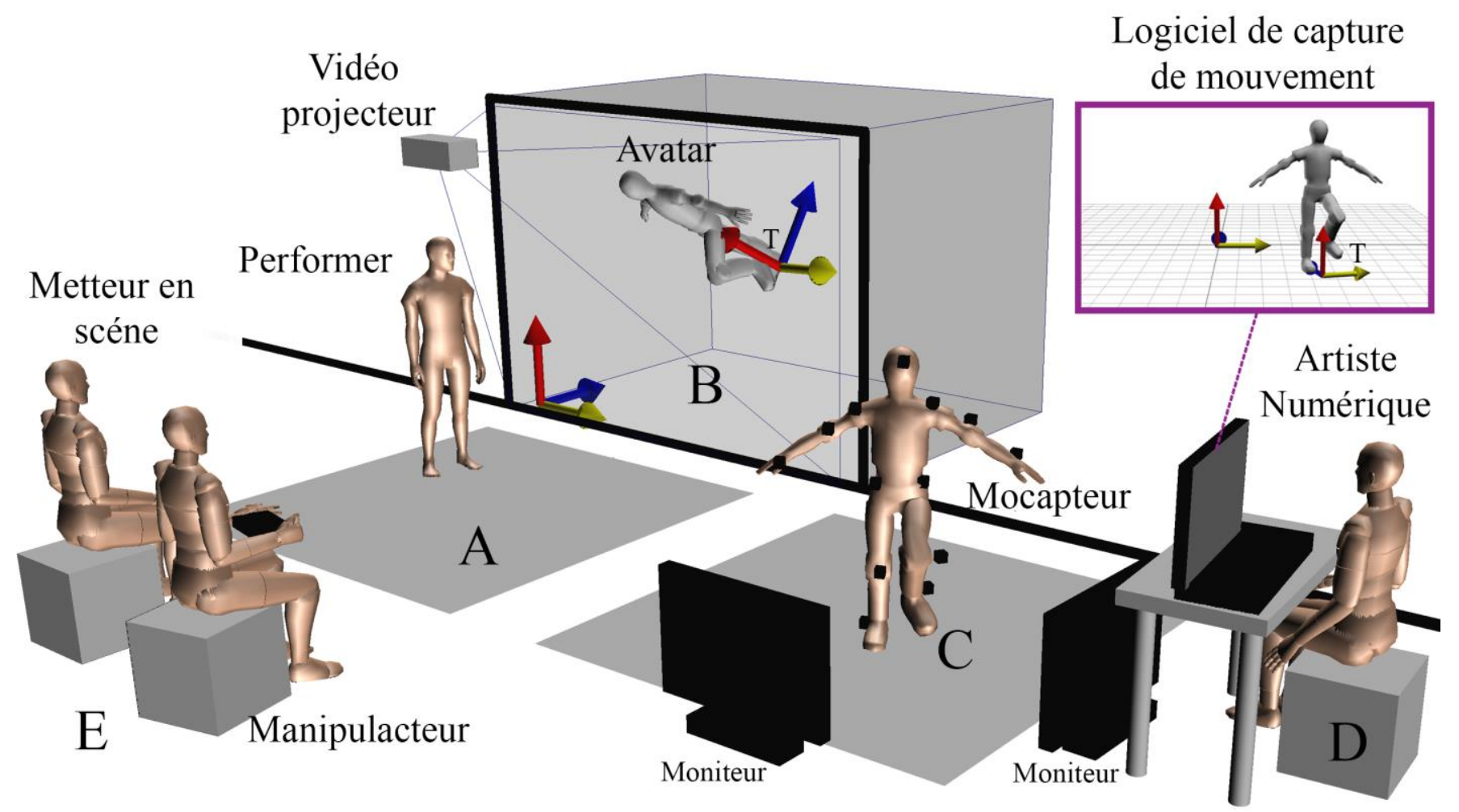

Figure 5. Dispositif AvatarStaging avec capture de mouvement inertielle.

AvatarStaging combine 5 espaces :

- Espace A : scène physique

- Espace B : scénographie numérique 3D projetée sous forme d'image en 2D

- Espace C : espace du mocapteur portant la combinaison de capture de mouvement

- Espace $\mathrm{D}$ : espace où l'artiste et le régisseur numérique construisent avatars et scénographie 3D

-Espace E : espace du public, du metteur en scène et du manipulacteur.

L'espace scénique procède de la réalité mixte dans le sens où il fait interagir les espaces $\mathrm{A}$ et $\mathrm{B}$. Les mouvements de l'avatar en $\mathrm{B}$ et les relations entre $\mathrm{A}$ et $\mathrm{B}$ dépendent des actions du mocapteur dans l'espace $\mathrm{C}$ et de la programmation numérique réalisée dans l'espace $\mathrm{D}$. La nature 3D de l'espace $\mathrm{B}$ implique l'utilisation d'un périphérique spécifique pour habiter les avatars.

Notre problématique est donc de transposer l'état de présence d'un corps humain à une sculpture matrice équipée d'un mécanisme d'actuation des mouvements. Nous parlerons ici de marionnette virtuelle pour décrire la nature de l'avatar généré par le mouvement de la sculpture matrice. Dans notre cas en effet, la source des mouvements est extérieure à la sculpture matrice et cette dernière n'a pas conscience de son environnement, l'origine de ces décisions est aussi extérieure. À noter qu'au contrôle du mocapteur peuvent s'ajouter les actions d'un manipulacteur qui utilisera une manette de jeu pour modifier certains paramètres du mouvement [GAG 18 a]. 


\section{Impact du changement de paradigme de la relation à une image 3D sur le jeu scénique}

\subsection{Du mocapteur à la marionnette virtuelle à travers une approche mimétique du jeu}

La transposition de l'état de présence du mocapteur à la marionnette virtuelle doit se décomposer en deux actions simultanées. D'un côté, le mocapteur transmet ses mouvements aux accéléromètres de la combinaison de capture, et d'un autre côté, après traduction algorithmique de ces mouvements en un squelette d'actuateurs applicable à une sculpture matrice, la marionnette virtuelle exécute les mouvements qui lui sont transmis. Nous sommes dans le cas d'un transfert de mouvement mimétique qui implique un engagement $\mathrm{du}$ corps entier $\mathrm{du}$ mocapteur faisant intervenir «le sixième sens » multisensoriel, le «sens du mouvement» décrit part Berthoz [BER 14]. Cet engagement du corps entier se réalise en effet dans un rapport mimétique avec la gestualité de la marionnette rendu possible par la similitude de construction du squelette des capteurs de la combinaison avec le squelette des actuateurs de la marionnette virtuelle (cf. figure 6).

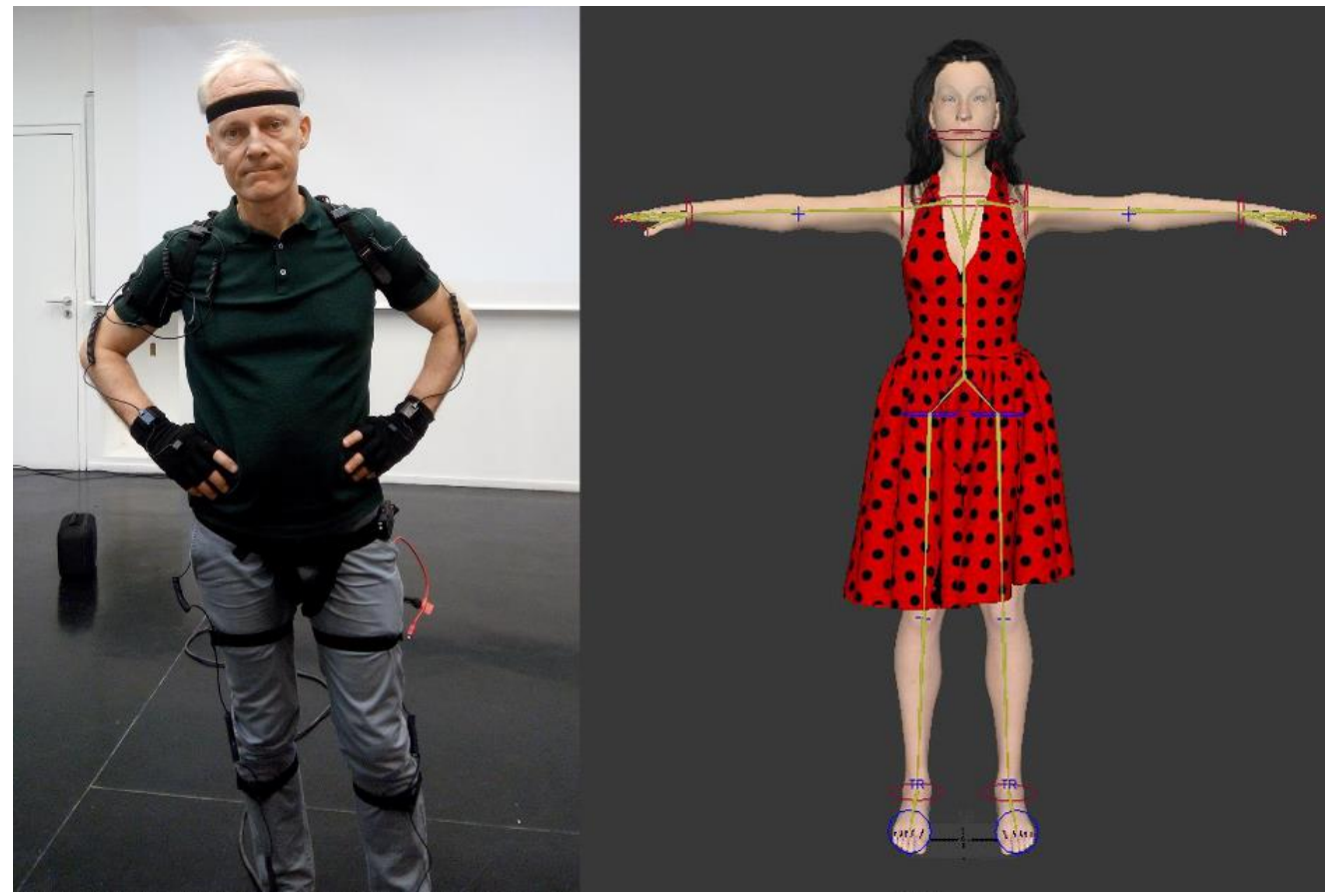

Figure 6. Combinaison de capteurs inertiels (gauche) et schéma des actuateurs 3D (droite)

Cette similitude est aussi présente dans le cas des combinaisons des dispositifs optiques utilisés par les studios de jeu vidéo pour capturer des mouvements très précis avec des batteries de caméras infrarouges repérant des réflecteurs posés directement sur le corps du mocapteur. En revanche, elle n'est pas véritablement présente dans le cas de la Kinect qui maintient un rapport vidéo dans la capture dans le sens où la Kinect ne prend qu'imparfaitement la profondeur du corps puisqu'une seule caméra infrarouge est utilisée.

Bien qu'elle semble naturelle, la maîtrise du rapport au squelette des capteurs nécessite un apprentissage spécifique. Le mocapteur doit en effet adopter une approche proprioceptive de ses mouvements, c'est-à-dire développer une perception consciente des parties de son corps qui portent les capteurs, ce qui est rendu plus facile par la présence de capteurs physiques dans les cas des dispositifs inertiels ou optiques. Cette approche proprioceptive permet une décomposition des mouvements directement adaptée aux capteurs, ce qui optimise l'algorithme de traduction des mouvements vers les actuateurs de la marionnette virtuelle. 


\subsection{Complémentarité possible par une approche dissemblable du jeu}

Cette approche est cependant plus difficile à adopter pour le manipulacteur qui n'agit souvent qu'à travers des gestes dissociés de leur conséquence sur le corps de l'avatar : par exemple, l'utilisation d'un joystick de manière horizontale pour faire tourner sur lui-même un avatar en sens horaire ou antihoraire selon qu'elle actionne le joystick à droite ou à gauche. Ce contrôle de la marionnette virtuelle est du même ordre que celui d'un animateur de jeu vidéo qui travaille sur les actuateurs de la sculpture matrice pas à pas sans une conscience intérieure des mouvements dessinés au moment de leur application sur l'avatar.

Ce phénomène entraîne un engagement haptique [WIT 12] que le manipulacteur acquiert par la pratique, et qui lui permet d'automatiser son geste à un niveau allant jusqu'au réflexe [CLA 03]. Il y a une analogie profonde avec ce que Kapandji évoque à propos de l'intégration du violon tenu en main par le violoniste dans son schéma corporel devenant ainsi un schéma corporel étendu [KAP 16]. Sohier a mené une réflexion pour le développement d'une interaction sensible au service du gameplay où il démontre de véritables possibilités artistiques exploitant les codes du «paradigme de la métaphore » et les transferts de mouvements dissemblables [SOH 16].

Le transfert de mouvement dissemblable exige ainsi une modification du schéma corporel et nécessite une réappropriation du corps virtuel induisant un travail plus complexe des mécanismes de contrôle, à la différence de la réappropriation du corps virtuel par le mocapteur dans le cas du transfert de mouvement mimétique.

\subsection{Multiplicité des processus d'incarnation}

On constate donc divers degrés d'incarnation dans le processus d'animation de la marionnette virtuelle. La prise de conscience de ce facteur d'incarnation a une influence sur la manière de jouer du mocapteur. On constate par ailleurs une influence de la nature visuelle de l'avatar sur les stratégies d'incarnation. Si la marionnette virtuelle a l'aspect d'une marionnette de théâtre traditionnel, le mocapteur aura tendance à articuler ses membres pour ressembler à une marionnette. Si la marionnette virtuelle présente une enveloppe corporelle moins squelettique et plus proche d'un corps avec une dynamique propre, le mocapteur aura tendance à articuler ses membres comme s'il se trouvait luimême à l'intérieur d'une mascotte (c'est-à-dire d'un costume de personnage comme l'on en trouve dans les parcs d'attraction).

«L'idée est que l'avatar qui porte la subjectivité à l'intérieur de l'univers numérique (qui, par le code universel de 0 et 1 , est d'un seul tenant, même si ses innombrables services et manifestations sont radicalement différents) est le lieu de fusion, de transformation et d'échange entre le subjectif et l'objectif, entre l'organique et le numérique. [...] À partir du moment où on pourra établir un contact direct et efficace entre pensée et écran ou réseau, la pensée pourra et devra vraisemblablement opérer une inversion radicale des polarités intérieurs-extérieur. C'est l'avatar qui lui servira de modèle. Car le passage par l'avatar permet de renverser les valeurs de l'intériorité et de l'extériorité. Du coup, mon corps devient un système d'occupation dans l'ensemble du vécu. » [KER 13] Cette vision de l'avatar fixe une ligne d'horizon. Elle porte l'intuition qu'un dialogue humain avec l'extériorité numérique pourra finalement conduire à une ouverture intérieure vers de nouveaux espaces.

\section{Cas d'usage : diriger simultanément un mocapteur et une marionnette virtuelle}

Les rapports spécifiques que le mocapteur et le manipulacteur établissent avec la marionnette virtuelle induisent une transformation des techniques de direction scénique habituellement utilisées sur une scène physique dans des scénographies non mixtes. Pour l'illustrer, nous nous appuierons sur un projet expérimental conduit sur les années 2017 et 2018 par une équipe d'artistes issue de l'université Paris 8 et Warwick University [GAG 18 b] [LAV 18]. 


\subsection{Description de la problématique}

La situation de mise en scène utilise une déclinaison du dispositif d'AvatarStaging qui fait jouer aux acteurs simultanément la fonction de mocapteur et de performeur devant l'espace 3D habité par les avatars contrôlés (cf. figure 7). C'est une situation scénographique particulièrement appréciée des metteurs en scène, probablement parce qu'elle permet de mettre en valeur le mocapteur dans sa fonction traditionnelle d'acteur. La difficulté qui en ressort pour les acteurs est de déployer leur présence scénique sur deux plans : celle de la scène physique avec leur propre corps, et celle de l'espace 3D avec le corps de l'avatar contrôlé.

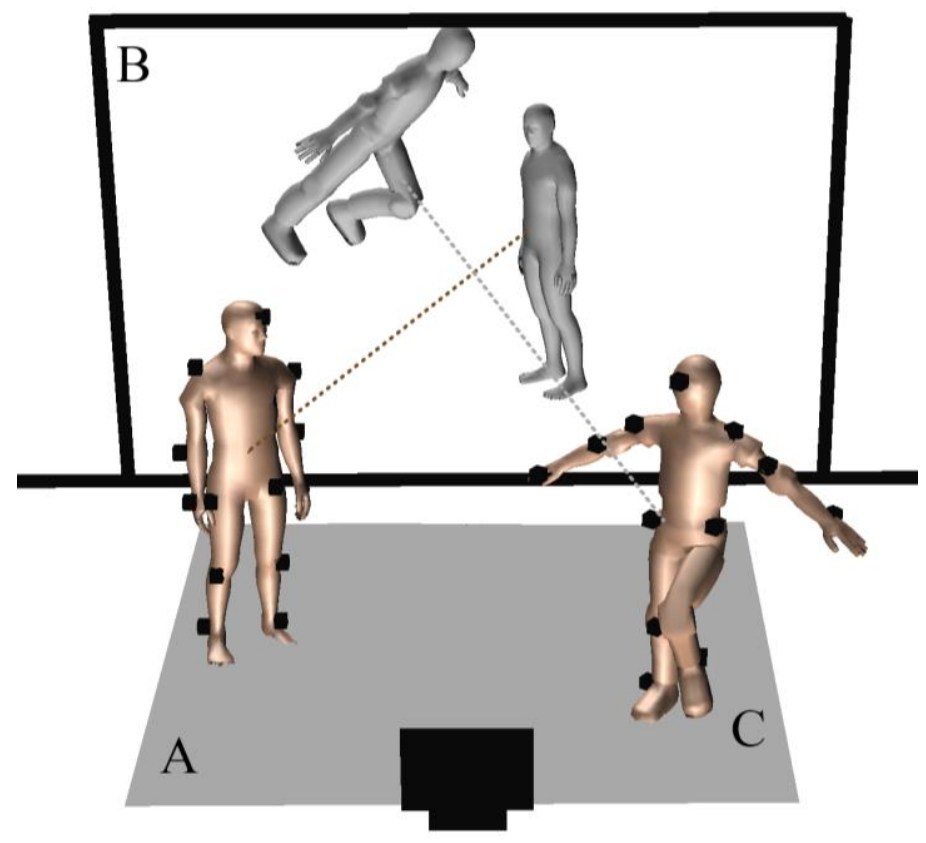

Figure 7. Performeurs ayant aussi une fonction de mocapteurs

Le metteur en scène occupe une place privilégiée qui lui permet d'observer simultanément les deux présences scéniques et de conseiller l'acteur sur ce qu'il pourrait faire pour obtenir les résultats souhaités dans la mise en scène. Il s'agit dans notre cas d'étude de faire en sorte que l'avatar blanc, contrôlé par l'actrice masquée, positionne précisément son corps dans une adresse à l'avatar bleu situé dans la voiture avec chauffeur. La figure 8 montre à gauche la scène sous un premier angle de vue (plan A), et à droite la même scène, quelques secondes plus tard, sous un angle de vue opposé (dans lequel on distingue clairement la voiture, un chauffeur et un passager à côté de l'avatar bleu - plan B).

Le metteur en scène peut diriger facilement les positions de l'actrice dans l'espace physique. Pour le contrôle de l'avatar dans l'espace 3D, nous utilisons un dispositif de projection de la sculpture matrice, animée par la capture des mouvements de la mocaptrice, disponible dans le module AKN_Regie que nous utilisons pour organiser le contrôle des avatars dans un espace 3D rendu avec un moteur de jeu. 


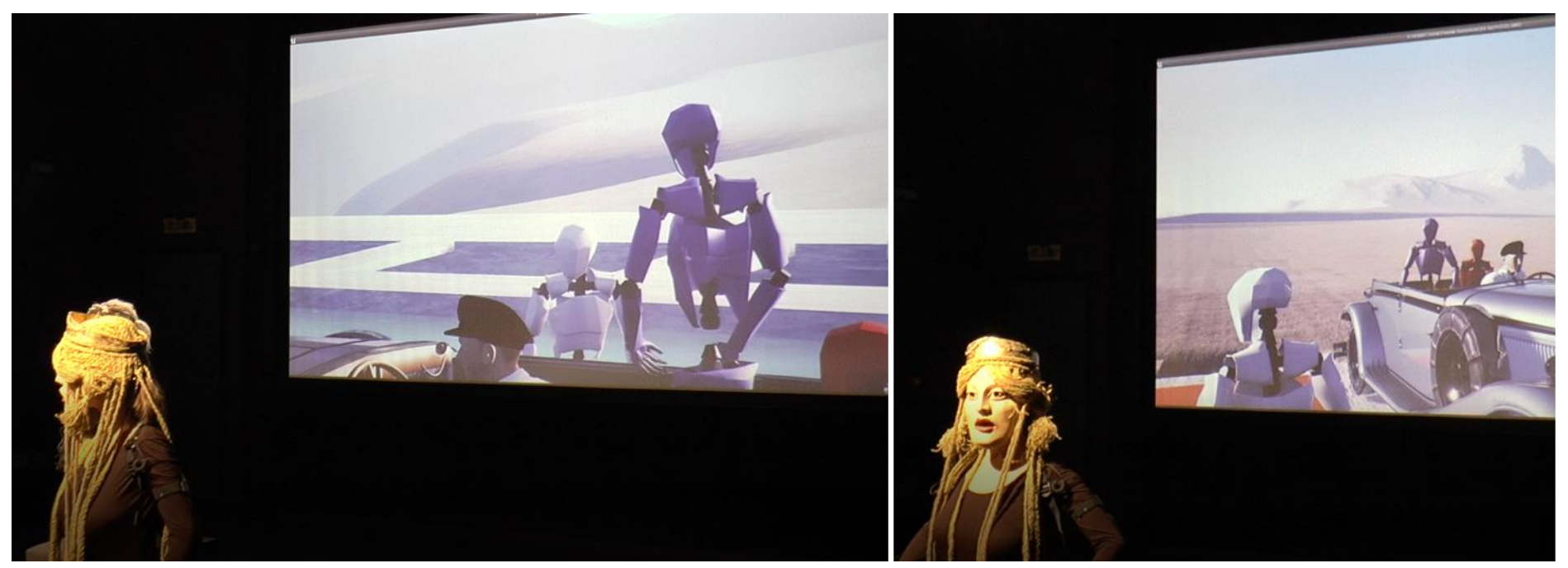

Figure 8. Adresses scéniques entre avatars blanc et bleu (plan A à gauche, $B$ à droite)

Le metteur en scène peut visualiser l'espace 3D et demander à son collaborateur s'occupant des questions de programmation numérique, que nous appellerons le régisseur numérique, de modifier les positions respectives des avatars et des éléments de décors. La figure 9 montre à gauche le metteur en scène discutant avec les mocaptrices et le régisseur numérique des projections qu'il souhaite réaliser. L'image à droite montre le rendu de l'espace 3D avec des avatars-figurants statiques et des caméras qui occupent les positions souhaitées (en l'occurrence GA48 pour l'avatar blanc, GA08 pour l'avatar bleu et GC06 pour la caméra correspondant au plan A).
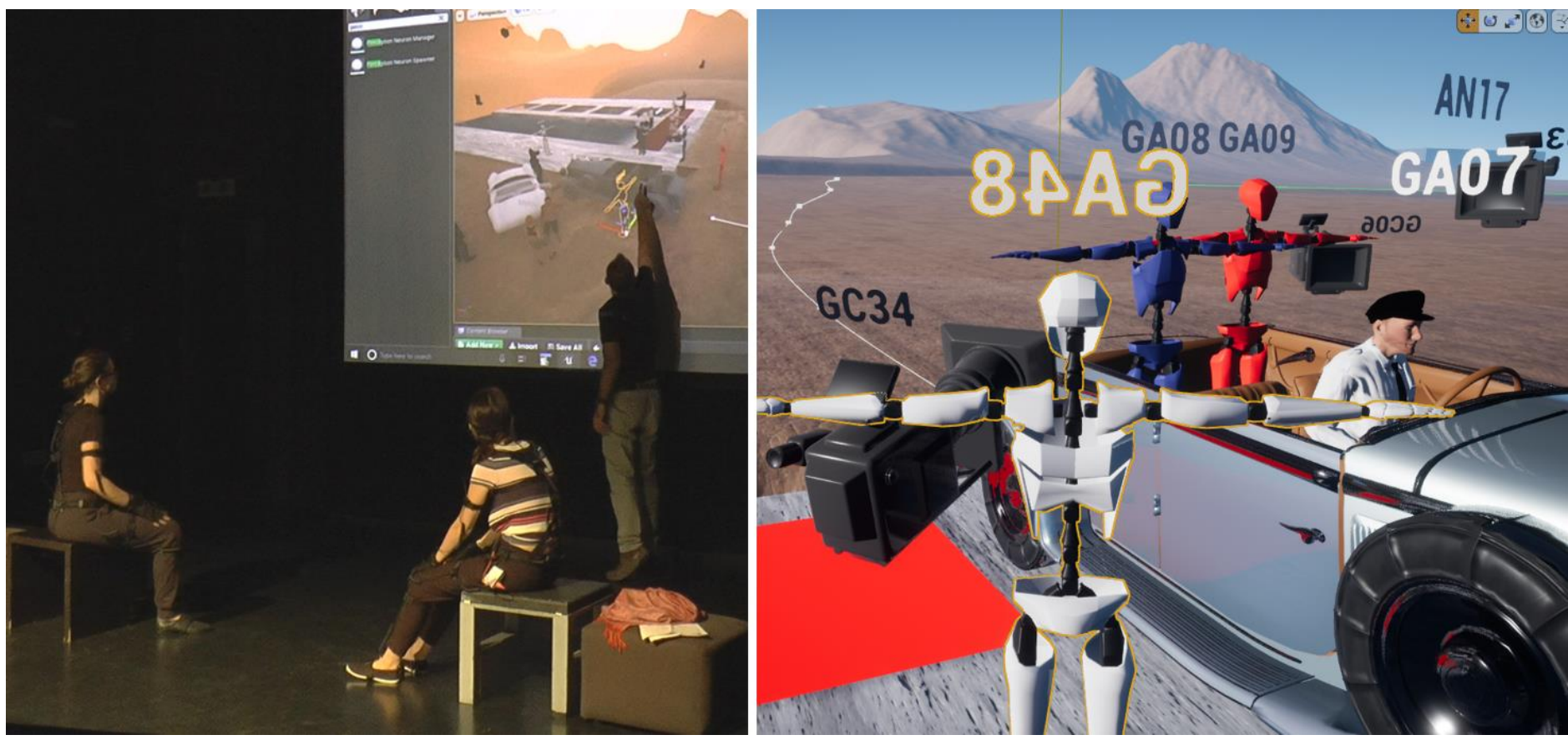

Figure 9. Ajustements de l'espace 3D (gauche) et positions 3D des avatars et des caméras (droite)

\subsection{Pièges du référentiel physique}

Les plans A et B sont les mêmes du point de vue de l'occupation de l'espace par les avatars, seule la position de la caméra change, avec un système de projection de la caméra sur des positions préétablies similaire à la technique employée pour les avatars. Le passage d'une image à l'autre se fait un avec système de liste d'actions à traiter que nous appelons «cue » (dont une traduction française serait «top »), et qui permet au régisseur numérique de lancer au moment désiré la projection de la caméra de rendu et de plusieurs avatars sur des positions précises (cf. figure 9 droite). 
Lors du déroulement de la répétition ou du spectacle, le régisseur numérique passe successivement de cue en cue selon ce qui a été décidé en mise en scène. Une page de cues (ou encore CueSheet, cf. figure 10 gauche) peut contenir des dizaines de cues. La cue 70 (cf. figure 10 droite) réalise par exemple le placement de la caméra dans la position GC06 pour obtenir le plan A, et des avatars blanc et rouge en position respective GA48 et GA08 (cf. figure 8 plan A).
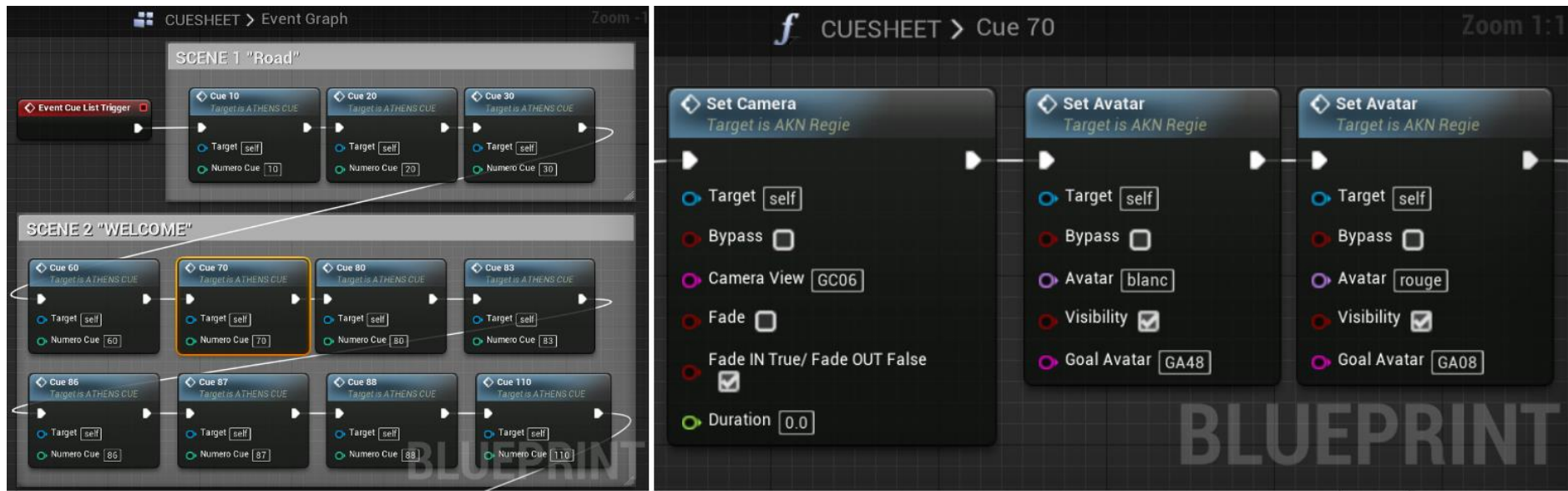

Figure 10. Page des cues du module AKN_Regie (gauche) et focus sur la cue 70 (droite)

Dans notre cas d'usage, la mocaptrice n'a pas possibilité, à cause de son masque, d'utiliser un retour visuel direct sur la scène 3D pour ajuster par elle-même la position de l'avatar qu'elle contrôle. Elle doit donc se reposer entièrement sur les instructions du metteur en scène. Pour organiser l'adresse scénique de l'avatar blanc, la première approche intuitive du metteur en scène est naturellement de contrôler la marionnette virtuelle comme s'il s'agissait d'une actrice traditionnelle. Il demande alors à la mocaptrice qui le contrôle de se placer dans l'espace physique sur une marque précise qui correspondrait à la place souhaitée de l'avatar blanc (cf. figure 11 gauche), et dans un deuxième temps, à ajuster l'adresse du regard vers l'avatar bleu en demandant à l'actrice de le suivre du regard jusqu'à ce que l'avatar prenne la position souhaitée (cf. figure 11 droite). C'est à la mocaptrice ensuite de mémoriser un détail dans la salle qu'elle devra regarder à nouveau au moment où elle sera sur la marque au sol établie précédemment.

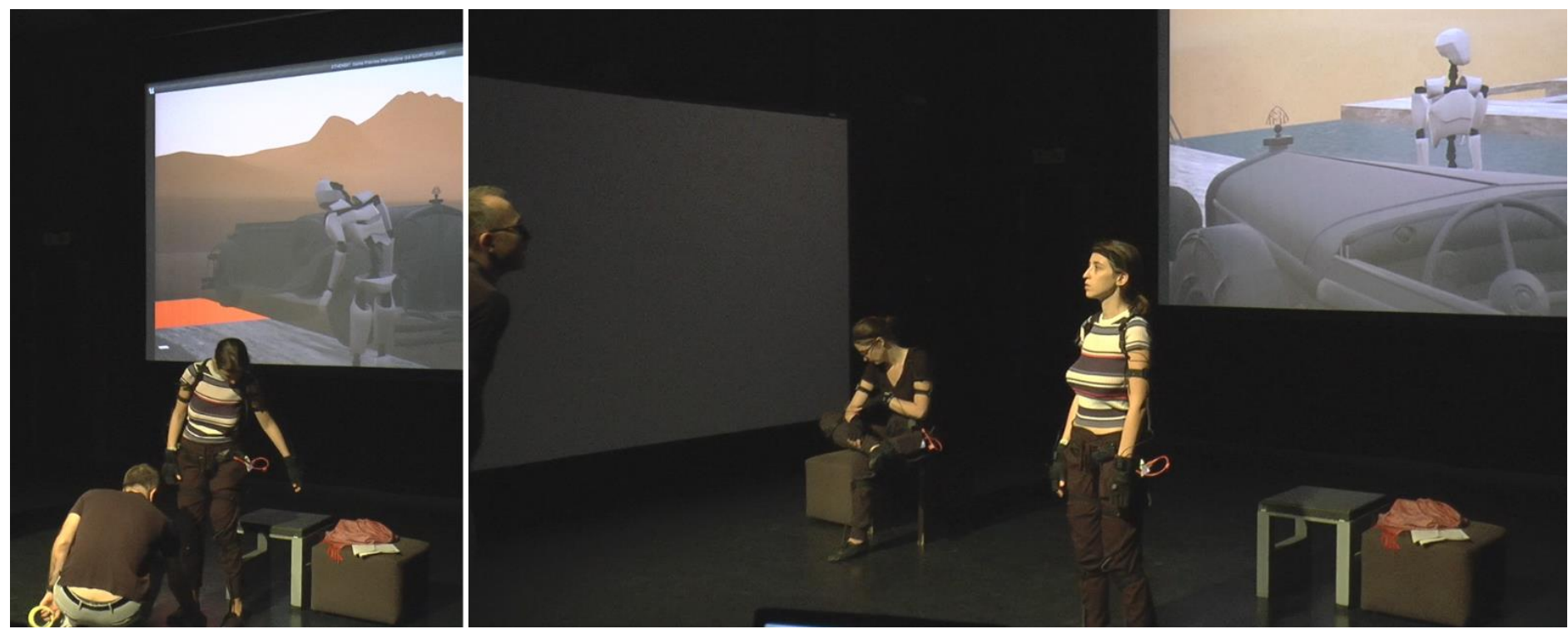

Figure 11. Ajustement de la position (gauche) et de la direction de regard (droite) dans l'espace physique

Ainsi, en contrôlant la mocaptrice, le metteur en scène espère diriger l'avatar blanc et obtenir l'adresse scénique souhaitée qui rendra plausible le dialogue entre les deux personnages virtuels (l'avatar bleu est contrôlé en l'occurrence par la seconde comédienne assise à gauche dans la figure 9). 
Pourtant, à chaque fois que la scène est jouée, et bien que la mocaptrice respecte scrupuleusement sa marque et sa direction de regard, l'avatar blanc ne regarde pas dans la bonne direction.

\subsection{Une solution introduisant la notion de position de référence virtuelle}

Une analyse de la programmation des cues permet de lever le mystère et conduit à un changement radical de perspective dans la manière de diriger les avatars pour le metteur en scène. Il se trouve en effet que la cue 70 (plan A) projette l'avatar sur une position précise, mais qu'aucune modification de position n'est opérée dans la cue suivante (plan B) qui ne contient qu'un changement pour la caméra. La comédienne effectue plusieurs mouvements dans l'espace après la cue $\mathrm{A}$ avant de prendre la position et la direction de regard nécessaire pour la cue $B$. Il se trouve qu'à chaque tentative d'ajustement de ces paramètres par le metteur en scène la position relative de l'avatar par rapport à sa position en A (GA48) a été chaque fois différente, ce qui fait que les réglages faits pour une position n'étaient plus valables pour une répétition suivante.

Il faut en effet abandonner la référence absolue à l'espace physique de jeu de l'actrice lorsque l'on veut diriger l'avatar qu'elle contrôle. La solution consiste à adopter la référence relative que constitue le corps même de l'actrice, et à projeter l'avatar sur une position numérique (GA48 par exemple) correspondant précisément à la position souhaitée au moment du changement de caméra. On appelle «position de référence virtuelle » cette correspondance d'une position physique de l'actrice en relation avec une position numérique préétablie. Pour organiser un regard ou un geste de la main concernant le haut du corps, il suffit que le metteur en scène demande à l'actrice, au moment du changement de cue, que sa position propre (ou encore relative) dans l'espace physique soit organisée spatialement en fonction de la position numérique donnée à l'avatar dans l'espace 3D.

Ainsi, indépendamment de sa position absolue dans l'espace scénique, au moment où la cue est lancée, l'actrice se remémorant la position de référence virtuelle correspondante peut contrôler l'adresse de l'avatar de manière précise. Le metteur en scène n'a donc pas besoin de lui donner des repères absolus avec une marque au sol et des directions de regard vers des points dans la salle, il lui faut seulement la diriger en relatif par rapport à son corps en relation à la position de départ de l'avatar.

\subsection{Implication pour le régisseur numérique et la mocaptrice}

L'adoption d'un système de positions de référence virtuelle implique un double travail pour le régisseur numérique. Il doit en effet veiller à chaque cue à ce que les positions numériques des avatars soient correctement programmées en fonction des attentes du metteur en scène et de la mocaptrice. En retour, il sera assuré d'obtenir la mise en place correcte des avatars dans le cadrage choisi pour la caméra, ce qui lui permet d'affiner l'écriture visuelle du spectacle en relation avec la scénographie 3D.

Par ailleurs, le régisseur numérique doit être vigilant lorsqu'il lance une nouvelle cue et se coordonner précisément avec ce qui a été établi d'un commun accord avec le metteur en scène et la mocaptrice. En effet, le déclenchement d'une cue impliquant une position de référence virtuelle suppose une position spécifique de la mocaptrice. Cela peut être calé sur du texte ou sur des mouvements, mais dans tous les cas, le déclenchement doit être précis. Cela est aussi valable pendant les répétitions. Par exemple, la reprise d'un moment entre deux cues impliquant des positions de référence n'est pas possible : le régisseur doit demander au metteur en scène de reprendre au moment de la première cue pour que la mocaptrice prenne sa position de référence. Il apparaît qu'une connaissance partagée par tous les protagonistes du système de positions de référence virtuelle est souhaitable pour le déroulement efficace et concentré des répétitions.

La difficulté pour la comédienne réside dans le fait de jouer en permanence sur deux plans de présence scénique. Elle doit en effet construire sa présence scénique en face des spectateurs de manière traditionnelle tout en adoptant régulièrement des positions qui assurent la présence scénique de l'avatar dans l'espace 3D. Le type de jeu par rapport à l'espace 3D est fortement corrélé à l'utilisation des 
possibilités de changement de position caméra et de mise en tension visuelle entre les avatars selon les cadres choisis. En comparant cette situation avec celle de l'acteur dans un dispositif de téléprésence (cf. 2.1.), on vérifie l'impact de la 3D sur le jeu. La mocaptrice doit en effet réellement faire un geste d'adresse scénique dans l'espace, correspondant à sa position de référence virtuelle. En téléprésence, l'acteur devait se contenter d'orienter son adresse selon sa position dans le cadre de la caméra, ce qui est plus abstrait et correspond rarement à une situation physique.

La possibilité d'explorer les vastes possibilités d'un monde 3D avec le langage spécifique du cinéma, dans une temporalité qui reste thêâtrale, et donc une performance en temps réel et non préenregistrée, conduit le jeu d'acteur vers de nouvelles conditions expressives. Cette manière de diffracter sa présence selon le langage visuel adopté implique la construction d'un sous-texte émotionnel et proprioceptif non intuitif. On retrouve ici du point de vue de l'acteur la même nécessité de se déprendre des approches traditionnelles que nous avions observé avec le metteur en scène essayant de diriger un avatar comme s'il s'agissait d'une personne physique.

\section{Perspectives}

\subsection{Vers une plausibilité de présence de la marionnette virtuelle}

Nous constatons donc que la transmission d'états de présence du mocapteur à l'avatar combine deux processus dans la manière de déployer le jeu scénique. Il y a tout d'abord un travail de compréhension du rapport à la structure de la marionnette virtuelle qui consiste à adapter une conscience proprioceptive intérieure du jeu en relation avec des potentialités d'actuation extérieures. Cela concerne la relation directe du mocapteur à l'avatar (cf. figure $12-1 \mathrm{er}$ cercle).

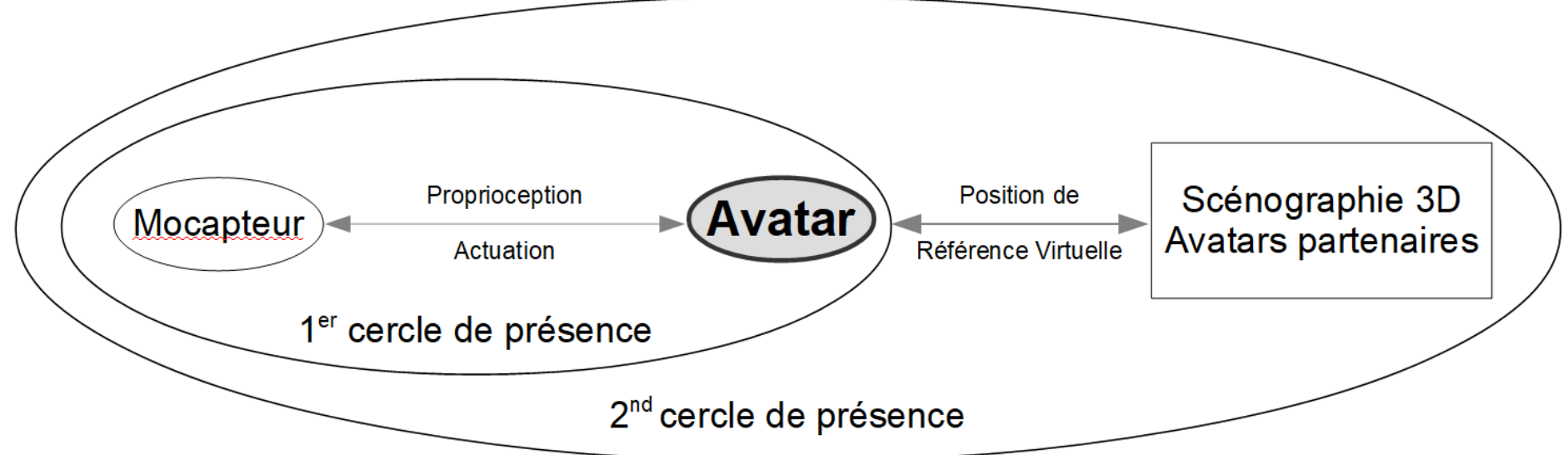

Figure 12. Cercles de déploiement des présences de l'avatar

Dans un second temps, le mocapteur doit prendre en compte la relation de l'avatar à son environnement 3D en mémorisant les positions de référence virtuelle qui ont été construites avec le metteur en scène et le régisseur numérique en fonction des nécessités de mise en scène et de la scénographie 3D (cf. figure $12-2$ nd cercle).

Cette approche du jeu permet d'améliorer la qualité de présence de l'avatar contrôlé dans l'espace 3D du point de vue du mocapteur. Elle concerne aussi les points de vue du metteur en scène, du manipulacteur et du régisseur numérique qui entretiennent des relations différentes avec l'avatar. Par exemple, la position extérieure du metteur en scène, combinée avec une double connaissance du travail de proprioception du mocapteur et du schéma d'actuation de la marionnette, permet de débloquer les difficultés au sein du premier cercle de présence en travaillant sur la compatibilité des mouvements proposés. Libéré du travail permanent d'introspection proprioceptive que doit réaliser le mocapteur, le metteur en scène est mieux à même de lui suggérer des combinaisons de gestes qui exploreront les 
capacités expressives propres à la marionnette virtuelle. De même, son rôle est déterminant dans la construction du second cercle de présence et l'établissement des positions de référence virtuelle.

\subsection{Multiplication des niveaux de jeu et empathie}

Dans le cas d'usage que nous avons traité en 5., la mocaptrice n'était pas en relation visuelle directe avec l'avatar qu'elle contrôlait. Cette situation nous a permis de décomposer précisément le processus de transmission d'une présence à l'avatar. Mais ces résultats restent valables quand le mocapteur dispose d'un retour vidéo sur l'avatar et son environnement 3D. Le mocapteur doit alors organiser les deux cercles de présence en juxtaposant non seulement une visualisation intérieure de son jeu à une visualisation extérieure du jeu transféré dans l'avatar, mais aussi se remémorer les positions de référence virtuelle qui garantissent sa relation à l'environnement 3D.

Nous avons démarré une exploration sur le mode de représentation de l'avatar dans les retours à destination du mocapteur, pour évaluer l'influence d'être représenté en mode miroir ou non, en nous inspirant des expérimentations menées par Alain Berthoz, Michel Bret et Marie-Hélène Tramus sur les interactions entre une funambule réelle et la funambule autonomel dans l'œuvre La Funambule virtuelle [THI 09]. Nous avons déjà eu plusieurs retours d'acteurs et d'actrices qui éprouvaient plus de difficulté à contrôler leur avatar en mode miroir, plutôt qu'en mode normal, qui induit une symétrie de rotation de l'avatar. Cela nous a conduit à envisager la notion de relation empathique du mocapteur à l'avatar, en écho aux expériences de Bavelas, cité par Jorland [JOR 04], qui induisent que «l'empathie établit une symétrie de rotation, la sympathie est une symétrie de réflexion». Dans la définition de Bavelas et Jorland, l'empathie consiste à « se mettre à la place d'autrui » et la sympathie «à s'identifier à autrui ». On constate en effet que le second cercle de présence invite le mocapteur à se projeter à la place de l'avatar pour donner sens à ses actions en fonction de l'environnement 3D.

Il faut préciser que les questions d'intériorité et d'extériorité dans la phase de contrôle temps réel de la marionnette virtuelle ne sont pas liées à la notion d'immersion 3D avec un casque de réalité virtuelle. Cette immersion permettrait effectivement de prendre le point de vue, au sens littéral, de la marionnette virtuelle sur son environnement 3D. Mais un acteir peut prendre ce point de vue en immersion tout en restant incapable d'habiter le corps de la marionnette virtuelle s'il ne fait pas tout d'abord le travail de correspondance proprioceptive avec le schéma d'actuation évoqué plus haut.

\subsection{Explorations de nouvelles techniques de jeu pour animer des avatars en temps réel}

Nous mesurons ainsi les conséquences du passage de la surface de la vidéo à la profondeur de l'espace 3D, et de la possibilité de transposer la présence d'un acteur dans une scénographie 3D avec un système wifi de captation des parties du corps en mouvement. Il est intéressant de noter que le passage à la 3D est corrélatif d'une exploration intérieure du corps et du mouvement. L'acteur ne doit plus seulement exposer son enveloppe corporelle à la caméra. Il doit explorer et reconstruire intérieurement la source de ces mouvements. Il s'agit d'un travail spécifique d'incarnation préalable à l'habitation de la sculpture matrice de l'avatar.

Ce travail est à prendre en compte dans la collaboration entre le mocapteur, le manipulacteur, le metteur en scène et le régisseur numérique. Il offre la possibilité d'explorer les potentialités d'une marionnette vivante (le mocapteur) qui consentirait à voir reconstruire de nouveaux fils entre les mains de partenaires (le manipulacteur ou le régisseur numérique). Nous avons établi quelques exercices pour mieux comprendre cette nouvelle configuration du jeu d'acteur et d'avatar [GAG $18 \mathrm{c}$ (cf. figure 13). 


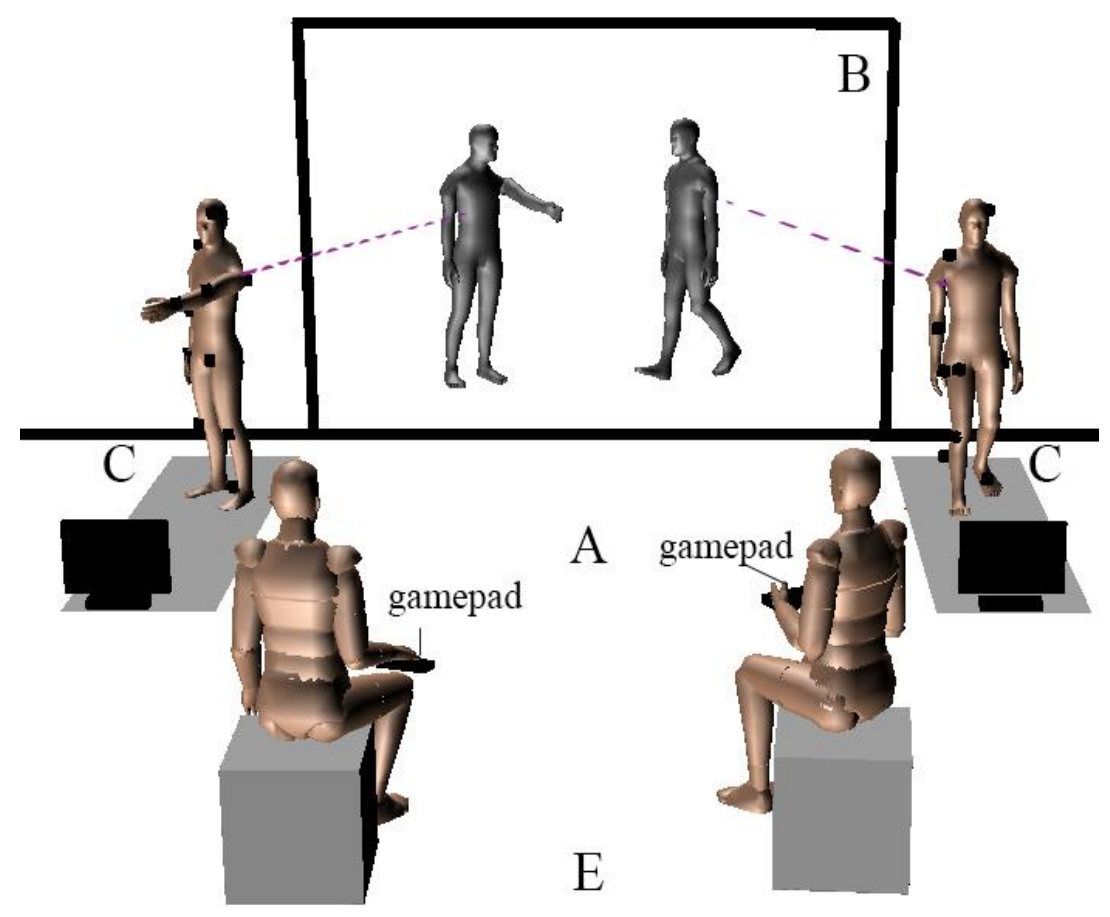

Figure 13. Exemple d'exercice de marche dans l'espace 3D

Par exemple, l'espace de jeu de le mocapteur est souvent circonscrit à un petit périmètre, il faut alors développer des techniques de jeu qui permettront de dépasser les limitations spatiales et de redonner toute sa liberté d'action à l'avatar. Nous avons notamment développé une approche qui intervient directement sur le mécanisme algorithmique d'actuation de la sculpture matrice. Lorsque le mocapteur a besoin de se repositionner dans l'espace sans que l'avatar ne bouge, il est possible de demander au manipulacteur d'enclencher un blocage de transmission des informations de mouvement aux membres inférieurs de la marionnette. Le manipulacteur peut bloquer l'avatar dans une position d'attente convenue avec le mocapteur, ce qui permet à ce dernier de se déplacer sans impact sur l'avatar et de reprendre cette position d'attente jusqu'à ce que le manipulacteur libère l'avatar qui peut alors réadopter les mouvements du mocapteur.

Ainsi s'ouvre un riche domaine expressif dans lequel il reste encore beaucoup de principes et de règles à découvrir.

\section{Bibliographie}

[AMA 13] Amato A., Perény E. (dir.), Les avatars jouables des mondes numériques. Théories, terrains et témoignages de pratiques interactives, Ed. Hermes Lavoisier, 2013

[BER 14] Berthoz A., Cours et travaux du Collège de France. Annuaire 113e année, Collège de France, Paris, avril 2014, p. $831-836$

[CLA 03] Clais, J.-B., et Roustan, M., 2003. « “Les jeux vidéo, c’est physique!” Réalité virtuelle et engagement du corps dans la pratique vidéoludique ». La pratique du jeu vidéo: réalité ou virtualité, 35-52.

[DIX 07] Dixon, S., Digital Performance, MIT Press, 2007

[FDI 11] Fdili Alaoui, S., Caramiaux, B. and Serrano, M., «From dance to touch: movement qualities for interaction design », CHI’11, 2011 may, pp.1465-1470

[FER 18] Féral, J. (dir.), L'Acteur face aux écrans. Corps en scène, Montpellier, L'Entretemps, coll. "Les voies de l'acteur », 2018, $448 \mathrm{p}$. 
[GAG 05 ] La Pluralité des Mondes. Texte Jacques Roubaud. Mise en scène et dispositif vidéo : Georges Gagneré. Jeu : Christophe Caustier. Composition musicale : Tom Mays. Programmation et vidéo : Pedro Soler et Christian Jacquemin (3D), avec la collaboration de Patrice Cros pour la réalisation des images. Lumière : Nathalie Perrier. Direction Technique, Régie vidéo numérique : Guy Levesque. Régie audio numérique : Olivier Pfeiffer. Diffusion : Emanuelle Walter. Production : didascalie.net. Création les 1 \& 2 Décembre 2005 à La Filature, Scène Nationale de Mulhouse.

[GAG 18 a ] Gagneré G., Plessiet C., Sohier R., «Interconnected virtual space and Theater. Practice as research on theater stage in the era of the network » in Challenges of the Internet of Things. Technology, Use, Ethics, Volume 7 (edited by I. Saleh, M. Ammi, S. Szoniecky), Wiley, 2018.

[GAG 18 b] Gagneré G., Plessiet P., A. Lavender, T. White, « Challenges of movement quality using motion capture in theater», in Proceedings of ACM MOCO conference, Genova, Italy, June 2018

[GAG 18 c] Gagneré G., C. Plessiet, «Experiencing avatar direction in low cost theatrical mixed reality setup », in Proceedings of ACM MOCO conference, Genova, Italy, June 2018

[JOR 04] Jorland, G., «L'empathie, histoire d'un concept». In L'empathie (Berthoz A. et Jorland G. dir.), Paris, O. Jacob, 2004, 308 p.

[KAP 16] , Kapandji A. 2016. «La main et le schéma corporel étendu ». Hand Surgery and Rehabilitation 35 (6). Elsevier Masson: 478-79. https://doi.org/10.1016/J.HANSUR.2016.10.171.

[KER 13] De Kerckhove, D., «L'occupation du virtuel. Relire Sartre » in Avatars, Personnages et Acteurs Virtuels (sous la direction de Bourassa, R.). Presse de l'Université du Québec. Ste-Foy, 2013, pp. 51-63

[LAV 18] Agamemnon Redux. Conception et mise en scène : Andy Lavender. Design numérique et direction technique : Tim White. Musique: Théo Semet. Jeu : Alexandra Beraldin et Cécile Roqué-Alsina. Lumière: Rob Batterbee. Programmation numérique: Georges Gagneré. Masques: Ferdinando Falossi. Création le 7 octobre 2018 à la Fondation Cacoyannis, Athènes, Grèce dans le cadre d'une invitation du projet Labex Arts $\mathrm{H} 2 \mathrm{H}$ « Scène Augmentée », dirigé par Erica Magris.

[PLE 18] Plessiet C., Gagneré G., Sohier R., « Avatar Staging: an evolution of a real time framework for theater based on an on-set previz technology », in Proceedings of the 2018 Virtual Reality International Conference. New York, NY, USA: ACM, 2018

[PLU 11] Pluta, I., L'Acteur et l'intermédialité. Les nouveaux enjeux pour l'interprète et la scène à l'ère technologique, Lausanne, L'Age d'homme, 2011, 395 pages.

[SAL 18] SAleh I. Ammi M. \& Szoniecky S. « Challenges of the Internet of Things : Technology, Use, Ethics », Co-publisher ISTE \& Wiley Editions, Londres, 2018, ISBN : 9781786303615.

[SAL 10] Salter, C., Entangled, MIT Press, 2010

[SLA 09] Slater, M., «Place illusion and plausibility can lead to realistic behaviour in immersive virtual environments », Philos Trans R Soc Lond B Biol Sci 364, 1535 (12 2009), pp. 3549-3557

[SOH 16] Sohier, R., 2016. «La dynamique du sensible dans la création de jeux vidéo d'art », Thèse de doctorat Esthetique, Sciences et Technologie des Arts sous la direction de Chu-Yin Chen, Université Paris 8

[STA 10] Staines, J., Boddington, G. Excited Atoms, OTM cultural mobility information dossier, 2010

[THI 09] Thirioux, B., Jorland, G., Bret, M., Tramus, M.-H., et Berthoz, A, 2009, « Walking on a line: a motor paradigm using rotation and reflection symmetry to study mental body transformations » in Brain and cognition 70 (2), Elsevier: 191-200.

[WIT 12] Witkowski, E., 2012, « On the digital playing field: How we "do sport" with networked computer games » in Games and Culture 7 (5), SAGE Publications, Los Angeles, CA: 349-74. https://doi.org/10.1177/1555412012454222 